Relations industrielles

Industrial Relations

\title{
Une expérience
}

Service d'hygiène industrielle

\section{Gérard Dion}

Volume 3, numéro 3, novembre 1947

URI : https://id.erudit.org/iderudit/1023576ar

DOI : https://doi.org/10.7202/1023576ar

Aller au sommaire du numéro

\section{Éditeur(s)}

Département des relations industrielles de l’Université Laval

ISSN

0034-379X (imprimé)

1703-8138 (numérique)

Découvrir la revue

Citer cet article

Dion, G. (1947). Une expérience : service d'hygiène industrielle. Relations

industrielles / Industrial Relations, 3(3), 41-42. https://doi.org/10.7202/1023576ar

Tous droits réservés (C Département des relations industrielles de l’Université Laval, 1947
Ce document est protégé par la loi sur le droit d'auteur. L'utilisation des services d'Érudit (y compris la reproduction) est assujettie à sa politique d'utilisation que vous pouvez consulter en ligne.

https://apropos.erudit.org/fr/usagers/politique-dutilisation/ 
existence and its life are commanded by an abstract consideration of rentability. ${ }^{2}$ It serves the employer, and not the community. In such an enterprise the capitalist is the animator of the structure, and in it he invests a part of his riches and the whole of his activities. If the labour contract which binds the worker to the enterprise respects the rules of ethics and human dignity, certain structural reforms may not be urgently required.

Non-personalized enterprise is made up of two main elements: the joint-stock company and the wage-earning. The world actually owes to the joint-stock company most of the great commercial and industrial progress that has characterized the last century. Among other things it has allowed the extension of the right of ownership to a great number of citizens and it may be said that it represents a trial at appropriation of enterprise by the community associated with a formula for comanagement. There is nothing essentially unjust in the wage contract binding together employers and employees within capitalist enterprise. Unfortunately, the capitalist economic regime has grown in an environment of economic liberalism and «the juridical institutions intended to foster the collaboration of the investments by dividing and limiting the risks involved have too frequently given occasion to the most reprehensible of abu-

(2) G. Desbuquois, P. Bigo, «Les réformes de l'entreprise et la pensée chrétienne », page 6 . ses,» according to the very works of Pope Pius XI. A « discretionary economic domination [has been left] in the hands of a few and those few are frequently not the owners, but only the trustees and directors of invested funds, which they administer at their good pleasure.»

In such a system, in which everything is directed towards profit, the employer's concern for his employees is subordinate to the interests of capital. In the joint-stock company, capital, an inert object, commands the worker, a human person. So that labour may be present at the meetings where its fate is being decided, and in order to comply with the legitimate aspirations of the working class which has become aware of its majority, structural reforms must be achieved, and institutions that will organize the representation of personnel with respect to management must be created.

\section{Conclusion:}

It is therefore permissible to believe that the best way to make a communitary reality out of enterprise especially out of large enterprise, is to modify somewhat the wage contract, whenever possible, «by introducing into it elements drawn from the contract of partnership as has already been tried in various ways to the not small gain both of the wage earners and of the employers. $\gg^{3}$

(3) Pope Prus XI, id. no. 72.

\section{Une expérience}

\section{SERVICE D'HYGIÈNE INDUSTRIELLE}

\section{Gérard DION}

A quelques reprises, dans le «Bulletin des relations industrielles », il a été question d'hygiène industrielle et des bienfaits de l'organisation d'un tel service dans les usines. Quelques-uns de nos lecteurs nous ont manifesté le désir de connaître des réalisations concrètes dans une petite entreprise. Des expériences ont été tentées ici et là. Nous croyons utile de reproduire ici, avec la bienveillante permission des parties concernées, le contrat passé entre le Dr Wilfrid LeBlond, professeur d'hygiène industrielle à notre Faculté, et Jos. Morneau
Inc., tel que nous le trouvons publié dans le journal d'usine L'ARBRE. ${ }^{1}$

1o. Le médecin s'engage à assurer la surveillance périodique de l'état sanitaire des locaux et lieux de travail ainsi que des annexes, avec rapport à l'employeur.

2o. Le médecin s'engage à faire l'examen médical de l'ouvrier suivant les modalités ainsi fixées :

(1) L'ARBRE, journal d'usine, Jos. Morneau Inc., octobre 1947, pp. 3 et 4. 
(a) l'examen de tous les employés se présentant à l'embauchage; (b) l'examen périodique annuel de tous les employés; ceci comporte l'examen physique, l'analyse du sang, l'examen des urines et une radiographie pulmonaire ou examen des poumons aux rayons-X; (c) examen en tout temps à la demande de l'employeur d'un ouvrier qui réclame pour maladie ou accident non causés par le travail; (d) l'examen de l'ouvrier pour apprécier s'il est apte au travail après une période d'incapacité due à un accident ou à la maladie; (e) l'examen périodique (au moins tous les 6 mois) des ouvriers engagés à des travaux comportant un risque déterminé pour leur santé ou leur sécurité.

Tous ces examens seront faits soit dans un local assigné à cette fin dans les bureaux de l'employeur, soit au bureau du médecin en ville ou à l’hôpital.

3o. Le médecin s'engage à assurer dans le meilleur esprit d'hygiène industrielle, la diffusion parmi les employés des notions d'hygiène générale: alimentation, vêtement, mesures de santé industrielle; propreté, régularité de la vie, prévention de la tuberculose, des maladies vénériennes, par des entretiens, des publications, des tracts, etc.

4o. Le médecin s'engage à assurer l'organisation et la surveillance du fonctionnement d'un poste de premiers soins aux blessés suivant les directives établies par le Ministère Fédéral de la Santé.

Toutes ces fonctions seront remplies à des heures déterminées chaque semaine, quant au nombre, par le nombre des employés, et quant à leur moment de la journée par entente entre le médecin et l'employeur.

Le présent engagement est pris pour et en considération de la somme de 24 sous par semaine par ouvrier ou employé figurant sur la liste de paie de l'employeur, payable au médecin le dernier jour ouvrable du mois. (contribution 50\% par l'employé, soit 0.12 et la balance par l'employeur.)

En vertu de quoi le médecin s'engage à ne faire aucune autre réclamation ni à l'employeur, ni aux employés pour les services précédemment énumérés.

L'employeur s'engage à mettre à la disposition de son service de santé, dès que les facilités du local le permettront, les espaces et le matériel nécessaire pour le fonctionnement intégral de son service de santé sur les lieux du travail même, et à suivre dans l'organisation matérielle de ce service à l'usine les plans et recommandations du Ministère Fédéral de la santé; section Hygiène industrielle à ce sujet.

Appendice I. - Traitement médico-chirurgical des employés atteints de maladie ou d'accident ne résultant pas de leur travail. Ces employés seront dirigés après examen, dans les locaux de la compagnie, ou au bureau du médecin, vers leur médecin de famille ou le médecin de leur choix pour traitement, et il est entendu que la responsabilité de l'employeur n'est aucunement engagée ni vis-à-vis l'employé pour le traitement ou la compensation de ces cas.

Appendice II. - Accidents du travail. Les cas d'accidents du travail seront traités suivant la Loi des accidents du travail, l'employeur se réservant le droit de faire examiner l'accidenté en cours de traitement et à la fin de la période d'incapacité totale temporaire, lorsque l'accidenté aura choisi un autre médecin que celui de la Compagnie pour le traitement de son accident.

Il est à noter que cette entreprise, dirigée par des jeunes patrons actifs, possède à son service une trentaine d'employés groupés dans un syndicat. Elle a aussi à son crédit d'autres réalisations sociales: un fonds de pension, une cantine, des repos intercalaires dans l'avant-midi et l'après-midi, un comité de coopération.

\section{SÉRIES DU BULLETIN}

Le Département des Relations industrielles dispose de quelques-unes des séries I et II de son Bulletin. Au premier volume, il manque toutefois le numéro 8.

On peut se procurer ces séries en s'adressant au secrétariat du Département, 2, rue l'Université, Québec.

Prix du volume: $\$ 1.50$. 